\title{
„Hauptsache das Kind ist gesund?“ - Ethische und rechtliche Fragen am Lebensanfang
}

\author{
Ruth Denkhaus $\cdot$ Julia Inthorn
}

Angenommen: 17. Dezember 2021

(C) Der/die Autor(en) 2022

Debatten zu ethischen Fragen am Lebensanfang sind international ebenso wie in Deutschland wohl etabliert. Mit zunehmenden medizinischen Möglichkeiten sowohl vor als auch nach der Geburt werden ethische Fragen aufgeworfen, bei denen die besondere Konstellation von (werdenden) Eltern und zukünftigen oder gerade geborenen Kindern berücksichtigt werden muss. Der Wunsch nach einem gesunden Kind steht dabei im Zentrum vieler Abwägungen. Genetische und andere Testmöglichkeiten vor oder während einer Schwangerschaft, die intensivmedizinische Versorgung Frühgeborener und in Zukunft möglicherweise auch Eingriffe in die menschliche Keimbahn dienen dem Ziel, die Gesundheit eines Kindes bestmöglich zu sichern oder Entscheidungen dazu treffen zu können. Während dieses Ziel einerseits breit akzeptiert ist, hat sich zugleich ein kritischer Diskurs herausgebildet, in dem Argumente wie die Ablehnung jeglicher Form von „Selektion“ oder das „Recht auf Nichtwissen“ eine zentrale Rolle spielen. Dabei zeigt sich häufig eine enge Verbindung zwischen der Bewertung neuer technischer Möglichkeiten und der Diskussion $\mathrm{zu}$ deren rechtlicher Regelung.

Jüngere technologische Weiterentwicklungen sind vor allem im Bereich der genetischen Diagnostik und Therapie zu verzeichnen: Next generation sequencing (NGS) und neue Testverfahren, die auf der Analyse zellfreier DNA basieren, verändern die Pränatal- und Präimplantationsdiagnostik, und mit der CRISPR/Cas9-Methode sind gezielte Eingriffe in das menschliche (Keimbahn-)Genom möglich geworden. Diese Technologien ermöglichen es, auf niedrigschwellige Weise umfassende genetische

Ruth Denkhaus $(\bowtie) \cdot$ Dr. Julia Inthorn

Zentrum für Gesundheitsethik (ZfG) an der Evangelischen Akademie Loccum,

Knochenhauerstr. 33, 30159 Hannover, Deutschland

E-Mail: ruth.denkhaus@evlka.de

Dr. Julia Inthorn

E-Mail: julia.inthorn@evlka.de 
Informationen zu generieren, deren Aussagekraft im Blick auf den Gesundheitszustand des zukünftigen Kindes jedoch häufig begrenzt ist. Daraus ergeben sich neue Fragen etwa im Blick auf etablierte Praktiken z. B. bei der Aufklärung zur Aussagekraft von nicht-invasiven Pränataltests (NIPT) oder im Umgang mit Nebenbefunden bei der Präimplantationsdiagnostik (PID). Auf einer eher theoretischen Ebene lässt sich zudem beobachten, wie die skizzierten Entwicklungen zu argumentativen Verschiebungen und Neueinschätzungen führen, z.B. wenn die PID im Vergleich zu Keimbahneingriffen plötzlich als die vorzugswürdige, weil weniger risikobehaftete reproduktive Option erscheint. Die begleitenden Diskussionen zur ethischen Bewertung und Einordnung einer Technologie sowie in vielen Fällen auch zu den rechtlichen Rahmenbedingungen zur Sicherung eines spezifischen Gesundheitsziels erhalten zum Teil große gesellschaftliche und mediale Aufmerksamkeit.

Dabei sortieren sich die Positionen vielfach entlang typischer Konfliktlinien. Besonders deutlich wird dies bei einem Blick auf weiter zurückliegende Debatten: Bei der Verabschiedung des Stammzellgesetzes lag der Fokus auf der Auseinandersetzung um den moralischen Status des menschlichen Embryos, beim Gendiagnostikgesetz formierte sich die Diskussion um das Recht auf Nichtwissen und beim Präimplantationsdiagnostikgesetz um die Ablehnung jeder Form von „Selektion“. Die Kombination aus medienwirksamer Zuspitzung, Reduktion komplexer Entscheidungen auf Grundsatzfragen und Selbstreferentialität dürfte mit dazu beigetragen haben, dass die Komplexität ethischer Fragen in der ärztlichen Beratung (angehender) Eltern zu den verschiedenen medizinischen Möglichkeiten in der Debatte in den Hintergrund geraten ist. Neben der systematischen Begleitung der mitunter sehr lebhaft geführten gesellschaftlichen Diskussion zu einzelnen Technologien ist die Fachdebatte daher aufgefordert, theoretische Positionen und begriffliche Differenzierungen weiterzuentwickeln sowie in der Vermittlung zwischen Theorie und Praxis zu einer verantwortlichen Entscheidungspraxis beizutragen.

Dabei ist einerseits zu berücksichtigen, dass ethische Fragen am Lebensanfang vor einem jeweils spezifischen gesellschaftlichen Hintergrund und mit Bezug auf einen kulturell geprägten normativen Horizont thematisiert werden. In der internationalen medizinethischen Literatur wird der Begriff der Selektion z. B. wesentlich unbefangener als in Deutschland und zum Teil sogar mit positiven Konnotationen verwendet - bis dahin, dass unter dem Stichwort ,procreative beneficience“ eine Pflicht zur Auswahl des ,,besten“ Kindes postuliert wird. Umgekehrt wird das Argument des „Rechts auf Nichtwissen“ bzw. ,right to an open future“, das dem Verbot spätmanifestierender Erbkrankheiten im Gendiagnostikgesetz zugrunde liegt, im angelsächsischen Raum seit einiger Zeit kritisch hinterfragt. In der Auseinandersetzung mit den entsprechenden Argumenten können in der deutschsprachigen Diskussion etablierte Positionen weiterentwickelt oder geschärft werden.

Darüber hinaus ist eine enge Anbindung an die in der klinischen Praxis gesammelten Erfahrungen zu den ethischen Herausforderungen bei Entscheidungen am Lebensanfang notwendig. Häufig zeigt sich erst in der konkreten Umsetzung eines Verfahrens, etwa im Nachgang zu gesetzgeberischen Grundsatzentscheidungen, welche ethischen Fragen und Probleme dadurch im klinischen Alltag aufgeworfen werden. Empirisch-ethische Hinweise aus der Versorgungspraxis können Anlass geben, einmal getroffene Regelungen kritisch zu hinterfragen und ggf. zu adaptieren. 
Gerade beim prä- und perinatalen Lebensschutz zeigt sich darüber hinaus immer wieder, dass Entscheidungen z.B. über das Therapieziel für Frühgeborene nicht der erwarteten Logik folgen und Eltern die Gesundheit und Lebensqualität eines (zukünftigen) Kindes im Kontext persönlicher Wertvorstellungen und ihrer familiären Lebensumstände deuten. Aus einer Analyse des praktischen Umgangs und der in diesem Kontext aufgeworfenen ethischen Fragestellungen erhalten etablierte Debatten Anregungen mit hoher Relevanz für Theorie und Praxis.

Das Themenheft nimmt das Ziel der bestmöglichen Gesundheit von (zukünftigen) Kindern als Ausgangspunkt und widmet sich davon ausgehend ethischen und rechtlichen Fragen zum Einsatz therapeutischer und diagnostischer Möglichkeiten am Lebensanfang. Vor dem Hintergrund der skizzierten Entwicklungen und Desiderate will es einen Beitrag zur Weiterentwicklung der Debatte in Deutschland leisten und aktuelle Grundlagen für eine verantwortliche Entscheidungsfindung in der Praxis bereitstellen.

Den Auftakt bilden drei Beiträge, die die dynamischen Entwicklungen im Bereich der vorgeburtlichen genetischen Diagnostik (PID und pränatale Diagnostik, PND) aufgreifen und sich dabei zugleich kritisch mit theoretischen Ansätzen aus der internationalen medizinethischen Diskussion auseinandersetzen (Denkhaus, RehmannSutter) bzw. Besonderheiten der deutschen Rechtslage thematisieren (Schmitz und Düwell).

Christoph Rehmann-Sutter nimmt die breite Einführung der NIPT und die Aussicht auf eine Ausweitung ihres Einsatzgebietes über die Detektion von Trisomien hinaus zum Anlass, um Stephen Wilkinsons Kategorisierung der PND als Mittel der „selektiven Reproduktion“ kritisch zu überprüfen. Dabei geht es ihm - anders als Wilkinson - nicht um die moralische oder rechtliche Zulässigkeit bestimmter diagnostischer Verfahren, sondern um die vorgelagerte Frage, wie wir die pränatale Diagnostik als emergente soziale Praxis verstehen und beschreiben können und ob der Begriff „Selektion“ in diesem Zusammenhang angemessen ist oder nicht. Rehmann-Sutter wendet sich damit zugleich gegen eine Engführung der ethischen Diskussion auf die Regulierungsperspektive. Ausgehend von Theodore Schatzkis Verständnis sozialer Praktiken als Verknüpfungen von Tun und Sagen und der darin enthaltenen Annahme, dass Benennungen nicht neutral sind, sondern dem, was wir tun, einen bestimmten Sinn verleihen, kritisiert er die Verwendung des Begriffs ,selektive Reproduktion“ im Zusammenhang mit Entscheidungen über einen Schwangerschaftsabbruch nach pränataler Diagnostik als sachlich unangemessen. Zentrales Argument dabei ist, dass zwar eine Entscheidung für oder gegen ein bestimmtes zukünftiges Kind, aber gerade keine Auswahlentscheidung stattfindet. Zudem hält er die Thematisierung der PND unter dem Label „,selektive Reproduktion“ für normativ problematisch, weil dabei wesentliche Dimensionen des moralischen Konfliktes, in dem sich die Betroffenen befinden, ausgeblendet werden.

Auch Ruth Denkhaus wendet sich in ihrem Beitrag mit Julian Savulescu einem Autor zu, der den Begriff der „Auswahl“ (selection) bewusst affirmativ verwendet und unter dem Schlagwort ,,procreative beneficence“ sogar eine Prima-faciePflicht zur Auswahl zukünftiger Kinder unter dem Gesichtspunkt des größtmöglichen zu erwartenden Wohlergehens postuliert. Sie diskutiert Savulescus Prinzip des 
prokreativen Wohltuns vor dem Hintergrund neuerer technologischer Entwicklungen im Bereich der PID wie der Gewinnung und Amplifikation von zellfreier DNA aus der Blastozoelflüssigkeit bzw. dem Kulturmedium und der Verwendung umfassender, NGS-basierter Testplattformen, die den routinemäßigen Einsatz der PID als Add-on zu einer (aus anderen Gründen indizierten) In-vitro-Fertilisation mittelfristig zu einer realistischen Option machen und damit auch der Frage nach einer möglichen moralischen Pflicht zur Inanspruchnahme einer PID neue Brisanz verleihen könnten. Gegenüber Savulescu vertritt Denkhaus die These, dass sein Prinzip des prokreativen Wohltuns keine überzeugende Grundlage für eine solche Pflicht bietet, weil es (in der Terminologie der philosophischen Debatte um das Parfit'sche Nicht-Identitäts-Problem) ein Verständnis von reproduktiver Verantwortung als ,unpersönlicher“", nicht am Wohlergehen eines bestimmten bzw. bestimmbaren Kindes orientierter Verantwortung voraussetzt. Gleichzeitig regt sie an, das Verhältnis von reproduktiver Verantwortung und elterlicher Verantwortung nicht nur im Blick auf die PID, sondern auch im Blick auf andere Optionen wie Keimbahneingriffe genauer zu reflektieren.

Dagmar Schmitz und Marcus Düwell befassen sich in ihrem Beitrag mit einer Besonderheit der deutschen Rechtslage: dem Verbot der pränatalen Diagnostik spätmanifestierender Erbkrankheiten im Gendiagnostikgesetz (§ 15 Abs. 2 GenDG), dessen normative und empirische Prämissen sie zunächst systematisch rekonstruieren, um sie dann einer umfassenden Überprüfung zu unterziehen. In theoretischkonzeptioneller Hinsicht befassen sie sich dabei vor allem mit der Frage, inwieweit ein mögliches Recht des zukünftigen Kindes auf Nichtwissen bzw. auf eine offene Zukunft bereits vor der Geburt verletzt werden kann. Hilfreiche Ressourcen und Argumentationsmuster für die Begründung von Rechten zukünftiger Kinder sind aus ihrer Sicht im Kontext des Menschenrechtsdiskurses bzw. der Diskussion über die Rechte zukünftiger Generationen zu finden. Mit Blick auf Fortschritte im Bereich pränataler Therapien machen sie geltend, dass bei der Entscheidung über die Zulässigkeit bzw. Durchführung pränataler Diagnostik nicht nur das Recht auf Nichtwissen, sondern auch das Gesundheitsinteresse des zukünftigen Kindes Berücksichtigung finden sollte. Gleichzeitig weisen sie darauf hin, dass eine isolierte Betrachtung der Testung auf spätmanifestierende Erbkrankheiten angesichts zunehmender genomdiagnostischer Möglichkeiten und der damit potenziell verbundenen Gefährdungen der informationellen Selbstbestimmung des zukünftigen Kindes nicht zielführend ist. Ihr Beitrag zeigt damit zugleich exemplarisch, wie rechtliche Regelungen in der Biomedizin sich als dysfunktional erweisen können, wenn sie nicht fortwährend im Licht neuerer Entwicklungen überprüft und ggf. revidiert werden.

Mit Unterschieden zwischen der Diskussion in Deutschland und in anderen Ländern - hier: Israel - befasst sich auch der Beitrag von Aviad E. Raz, Tamar NovKlaiman, Yael Hashiloni-Dolev, Hannes Foth, Christina Schües und Christoph Rehmann-Sutter. Die Autor*innen untersuchen dazu exemplarisch die Regulierung und mediale Thematisierung der PID zur HLA-Typisierung (HLA-PID) sowie der NIPT. Dabei arbeiten sie u. a. heraus, dass sich die Situation deutlich nuancierter darstellt, wenn man nicht nur die HLA-PID betrachtet, bei der die jeweiligen Regulierungen und gesellschaftlichen Haltungen tatsächlich stark divergieren, sondern mehrere Verfahren zusammen in den Blick nimmt. Gegenüber der älteren These von Yael 
Hashiloni-Dolev und Shiri Shkedi, die die Differenzen im Umgang mit der HLAPID auf unterschiedliche kulturelle Auffassungen von Familie und den Beziehungen der Familienmitglieder zueinander zurückführen, betonen Raz et al. die Notwendigkeit, unterschiedliche kulturelle Faktoren und normative Arrangements in ihrem Zusammenspiel zu betrachten, statt einen einzigen, übergreifenden Erklärungsansatz heranzuziehen. So weisen sie u. a. darauf hin, dass neben dem jeweiligen Verständnis von Familienbeziehungen auch unterschiedliche Einschätzungen zur jeweiligen Schutzwürdigkeit von extra- und intrakorporalen Embryonen bzw. Föten in die nationalen Regelungsansätze hineinspielen. Insgesamt plädieren Raz et al. dafür, das Augenmerk in Zukunft stärker auf Mechanismen der Routinisierung und die Art, wie sie mit Praktiken der Entscheidungsfindung verknüpft sind, zu lenken.

Während die Beiträge von Rehmann-Sutter, Denkhaus, Schmitz und Düwell und Raz et al. die vorgeburtliche Situation im Blick haben, befassen sich André Kidszun und Brian Carter mit Informations- und Unterstützungsbedürfnissen von Eltern extrem unreifer Frühgeborener. Beide stützen sich für ihre Überlegungen auf die Ergebnisse einer an der Universitätsmedizin Mainz durchgeführten empirischen Studie, die darauf hinweisen, dass prognostische Aussagen zur Wahrscheinlichkeit verschiedener Outcomes von nachgeordneter Bedeutung für die Behandlungspräferenzen der Eltern sind (Kidszun et al. 2020), und nehmen diesen Befund zum Anlass, um grundsätzlicher nach Faktoren und Kriterien gelingender Entscheidungsfindung im Grenzbereich der Lebensfähigkeit zu fragen.

André Kidszun bietet zunächst einen Überblick über die Herausforderungen, die sich bei der Präsentation und Interpretation medizinischer Fakten im Aufklärungsgespräch mit Eltern stellen, um dann sein eigenes Verständnis von Entscheidungsunterstützung als Rahmenmodell der gemeinsamen Entscheidungsfindung zu skizzieren. Angesichts der Besonderheiten von Entscheidungen über die Behandlung extrem unreifer Frühgeborener an der Grenze zur Lebensfähigkeit wie z.B. der enormen prognostischen Unsicherheit und der Vulnerabilität der Eltern plädiert er dafür, die Entscheidung weder einfach den Eltern zu überlassen noch sie ihnen abzunehmen, sondern die Eltern gezielt dabei zu unterstützen, eine Entscheidung im Einklang mit ihren Werten und Überzeugungen zu treffen. Ärzt*innen sollten nach Kidszun aktiv explorieren, welche Rolle die Eltern im Entscheidungsprozess einnehmen wollen, und den Prozess entsprechend strukturieren. Dabei sollten vor allem die individuellen Erfahrungen, Werte und Ziele der Eltern zur Sprache kommen und von den behandelnden Ärzt*innen im Zweifelsfalle auch aktiv eruiert werden. Die Grenze zwischen Entscheidungsunterstützung, die autonomiewahrend und -fördernd wirkt, und paternalistischer Einmischung kann nach Kidszun am besten gewahrt werden, wenn der jeweilige Grad der Unterstützung selbst transparent gemacht wird.

Brian S. Carter ergänzt in seinem Kommentar die Überlegungen von Kidszun zu einer an den Bedürfnissen der Eltern ausgerichteten ärztlichen Beratung bei Behandlungsentscheidungen im Grenzbereich der Lebensfähigkeit aus der Perspektive eines US-amerikanischen Neonatologen und Bioethikers. Sein Fokus liegt dabei besonders auf der Bedeutung von Vertrauen als Grundlage einer tragfähigen Arzt-PatientenBeziehung. Für die Präsentation medizinischer Fakten und probabilistischer Aussagen zu möglichen Outcomes schlägt er einen stärker narrativ ausgerichteten Ansatz vor und benennt mit dem Stichwort ,[minimization of] decisional regret“ expli- 
zit ein Kriterium gelungener Entscheidungsfindungsprozesse, das vor allem auf die subjektive Entscheidungszufriedenheit zielt.

Die in diesem Heft versammelten Beiträge sind im weiteren Kontext der Arbeit der Arbeitsgruppe (AG) „Ethische Fragen am Lebensanfang“ in der Akademie für Ethik in der Medizin entstanden. Sie entwickeln themenbezogene Diskussionen der AG weiter (Denkhaus, Schmitz und Düwell), sind aus Forschungsprojekten von AGMitgliedern entstanden (Kidszun, Raz et al., Rehmann-Sutter) oder kommentieren die Ergebnisse dieser Projekte (Carter). Alle Beiträge zeigen dabei, wie sich in der spezifischen Verbindung aus gesellschaftlicher Diskussion und ethischem Fachdiskurs sowie zwischen Theorie und Praxis Fragestellungen und Argumentationen weiterentwickeln und welche Rolle das Ziel der Gesundheit eines (zukünftigen) Kindes dabei spielt.

Danksagung Wir danken allen Autor*innen für ihre Beiträge und die vertrauensvolle Zusammenarbeit. Den externen Gutachter*innen danken wir für konstruktive Hinweise zu den Beiträgen. Wir danken der Schriftleitung, dass dieses Themenheft ermöglicht wurde, Dieter Birnbacher und Ralf J. Jox für die Begleitung auf dem Weg der Veröffentlichung und besonders Andrea Kähling für ihre redaktionelle und organisatorische Arbeit.

Funding Open Access funding enabled and organized by Projekt DEAL.

Open Access Dieser Artikel wird unter der Creative Commons Namensnennung 4.0 International Lizenz veröffentlicht, welche die Nutzung, Vervielfältigung, Bearbeitung, Verbreitung und Wiedergabe in jeglichem Medium und Format erlaubt, sofern Sie den/die ursprünglichen Autor(en) und die Quelle ordnungsgemäß nennen, einen Link zur Creative Commons Lizenz beifügen und angeben, ob Änderungen vorgenommen wurden.

Die in diesem Artikel enthaltenen Bilder und sonstiges Drittmaterial unterliegen ebenfalls der genannten Creative Commons Lizenz, sofern sich aus der Abbildungslegende nichts anderes ergibt. Sofern das betreffende Material nicht unter der genannten Creative Commons Lizenz steht und die betreffende Handlung nicht nach gesetzlichen Vorschriften erlaubt ist, ist für die oben aufgeführten Weiterverwendungen des Materials die Einwilligung des jeweiligen Rechteinhabers einzuholen.

Weitere Details zur Lizenz entnehmen Sie bitte der Lizenzinformation auf http://creativecommons.org/ licenses/by/4.0/deed.de.

Interessenkonflikt R. Denkhaus und J. Inthorn geben an, dass kein Interessenkonflikt besteht.

\section{Literatur}

Kidszun A, Matheisl D, Tippmann S et al (2020) Effect of neonatal outcome estimates on decisionmaking preferences of mothers facing preterm birth: a randomized clinical trial. JAMA Pediatr 174(7):721-722. https://doi.org/10.1001/jamapediatrics.2020.0235 